\title{
Informatics Strategies for Large-Scale Novel Cross-Linking
}

\section{Analysis}

\author{
Gordon A. Anderson $_{\star}^{\dagger}$, Nikola Tolic ${ }^{\dagger}$, Xiaoting Tang ${ }^{\ddagger}$, Chunxiang Zheng ${ }^{\ddagger}$, and James E. \\ Bruce $^{*}, \ddagger$ \\ Environmental Molecular Sciences Laboratory (EMSL), Pacific Northwest National Laboratory \\ (PNNL), Richland, Washington 99352, and Department of Chemistry, Washington State University \\ (WSU), Pullman, Washington 99164-4630
}

\begin{abstract}
The detection of protein interactions in biological systems represents a significant challenge for today's technology. Chemical cross-linking provides the potential to impart new chemical bonds in a complex system that result in mass changes in a set of tryptic peptides detected by mass spectrometry. However, system complexity and cross-linking product heterogeneity have precluded widespread chemical cross-linking use for large-scale identification of protein-protein interactions. The development of mass spectrometry identifiable cross-linkers called protein interaction reporters (PIRs) has enabled on-cell chemical cross-linking experiments with product type differentiation. However, the complex datasets resultant from PIR experiments demand new informatics capabilities to allow interpretation. This manuscript details our efforts to develop such capabilities and describes the program $X$-links, which allows PIR product type differentiation. Furthermore, we also present the results from Monte Carlo simulation of PIR-type experiments to provide false discovery rate estimates for the PIR product type identification through observed precursor and released peptide masses. Our simulations also provide peptide identification calculations based on accurate masses and database complexity that can provide an estimation of false discovery rates for peptide identification. Overall, the calculations show a low rate of false discovery of PIR product types due to random mass matching of approximately $12 \%$ with $10 \mathrm{ppm}$ mass measurement accuracy and spectral complexity resulting from 100 peptides. In addition, consideration of a reduced database resulting from stage 1 analysis of Shewanella oneidensis MR-1 containing 367 proteins resulted in a significant reduction of expected identification false discovery rate estimation compared to that from the entire Shewanella oneidensis MR-1 proteome.
\end{abstract}

\section{Introduction}

The growth of high-speed whole genome sequencing has fueled significant demand to assign genes and determine global functionality of the resulting proteins. ${ }^{1}$ The fields of proteomics research, mass spectrometry, antibody arrays, and chemical and molecular biology methods have become the focus of significant research efforts to help map large-scale functionality of proteins. Among the many challenges inherent in today's proteomics research, a significant unmet demand is related to the ability to measure and identify protein-protein interactions in cells, tissues, or biological fluids. In part, this is due to the fact that protein interactions

\footnotetext{
* To whom correspondence should be addressed. James E. Bruce, Department of Chemistry, Washington State University, Pullman, Washington 99164-4630; Tel, 509-335-2116; Fax, 509-335-8867; E-mail, james_bruce@ wsu.edu.

†Pacific Northwest National Laboratory.

\$Washington State University.

Supporting Information Available: Figures 1-4. This material is available free of charge via the Internet at http://pubs.acs.org.
} 
themselves present no common physical parameter that can be used for their detection other than the requisite close proximity of interacting partners. Thus, analytically, this is a difficult feature to map among many different protein-protein interactions within the confines of a cellular system. Despite these challenges, the protein interaction environment has a major impact on protein function, and large-scale protein interaction information is a prerequisite to comprehensive understanding of biological organisms on a systems-level. Yeast two-hybrid and coimmunoprecipitation 2,3 methods have become a primary means to acquire large-scale protein-protein interaction information. To date, these methods have produced the large majority of interaction information, but do not provide optimal solutions for large-scale protein interaction mapping in cells.

Conceptually at least, chemical cross-linking provides a route to impart new covalent bonds in a biological system such that protein-protein interaction information can be determined through subsequent mass spectrometry. ${ }^{4-10}$ The close spatial location of interacting proteins that facilitate chemical cross-linking can result in observation of masses of cross-linked peptides from both proteins. In general, these observed cross-linked product masses do not allow identification of interacting proteins due to the overwhelmingly large number of possible combinations that can give rise to the observed masses, even for fairly simple biological systems. When considered from the point of view of a classic bottom-up proteomics paradigm of peptide identification, the nonspecific database search based on the measured mass of crosslinked products becomes an $N^{211}$ problem, where $N$ is the possible number of tryptic peptides from the whole proteome. Although high resolution, accurate mass measurement methods hold great promise for high throughput proteomics experiments, even state-of-the-art mass spectrometry is insufficient for unambiguous identification of protein-protein interactions from conventional cross-linked peptide analysis. In other words, from the analysis of largescale chemical cross-linking samples, very few if any observed cross-linked product masses are unique and allow unambiguous protein interaction identification. Furthermore, MS/MS methodologies applied to cross-linked peptides can result in the simultaneous observation of a combination of fragments from both peptides, as well as the cross-linker with spectral complexity that can preclude any useful interpretation. As a result, most chemical cross-linking strategies have shown greatest usage in recent years as useful tools for the structural characterization of purified protein complexes. 8,9

We have developed and applied a novel strategy that involves chemical cross-linking for protein-protein interaction identification from live cells that is based on a novel class of chemical cross-linkers we refer to as protein interaction reporters or PIRs. ${ }^{8}$ PIRs provide unique capabilities for protein-protein interaction identification from cells because the PIRs are engineered with mass spectrometry-cleavable bonds that can be fragmented with high selectivity. The PIR strategy effectively encodes the cross-linkers with information that can be retrieved during the analysis to allow identification of proteins, differentiation of cross-linked product types, and elucidation of the regions of protein that are in close proximity. With this new chemical biology method, new informatics demands have arisen to allow large-scale identification of PIR product types in complex mass spectrometry data streams. The large quantity of complex data generated in PIR proteomics experiments quickly overwhelms the ability to manually inspect data and efficient application of the PIR technology ultimately depends on new informatics capabilities. Therefore, the focus of the present paper is to provide a detailed description of the processing central to PIR datasets and description of the program $X$-links that we have developed to provide this informatics capability. Furthermore, we also present results from Monte Carlo simulations of false discovery rates of PIR differentiation due to random matching between peptide masses. Our data also illustrate the feasibility in the identification of peptides with accurate mass and other PIR strategy constraints as a function of database complexity. 


\section{Methods}

The structures, mass spectrometry fragmentation properties, performance of the PIR strategy with known purified complexes, and identification of PIR-labeled proteins from Shewanella oneidensis are presented elsewhere. ${ }^{10,12}$ All simulations of false discovery rates for PIR relationship differentiation and accurate peptide mass-based protein identification were carried out using the $X$-links application and macros written in Microsoft Excel. The term "false discovery" is used here to indicate the identification of peptide masses other than those correct masses (previously chosen for precursor mass calculation) that sum with reporter mass to match expected values of the defined precursor. The application $X$-links that was used to analyze the experimental PIR datasets and to help identify PIR product relationships was also developed with the use of Microsoft's Visual Basic programming environment. The genome of Shewanella oneidensis MR-1 was downloaded from The Institute for Genome Research (TIGR) and was used with the $X$-links application to calculate all possible tryptic peptides with variable (up to one) allowed missed cleavages in the mass range from 500 to $5000 \mathrm{Da}$.

The example cross-linker data set was generated from a sample prepared from bradykinin and neurotensin purchased from Sigma chemical Co (St. Louis, MO). A stock solution of neurotensin was prepared in dry DMF at a concentration of $1 \mathrm{mM}$. The stock solution of bradykinin was prepared in water at a concentration of $10 \mathrm{mM}$. The stock solution of crosslinker was prepared in dry DMF at a concentration of $1 \mathrm{mM}$. The peptides were mixed in a 1:1 ratio and dried in a vacuum centrifuge prior to use. Next, an aliquot of the cross-linker solution that contained 0.5 molar equiv of cross-linker was added to the dried peptides. The reaction mixture was vortexed and incubated at room temperature overnight on a thermo-mixer (Eppendorf, Westbury, NY) at 900 RPM. After incubation, the reaction was quenched with 10 $\mu \mathrm{L}$ of $200 \mathrm{mM}$ ammonium bicarbonate solution. The solvent and salt were removed in vacuum centrifuge at $50{ }^{\circ} \mathrm{C}$. The dried reaction products were then dissolved in $49 \%$ methanol, $49 \%$ water, and $2 \%$ acetic acid.

This sample analysis was performed on a Bruker 7T FTICR mass spectrometer. The ion accumulation period was $0.5 \mathrm{~s}$. An experiment was designed and used to alternatively apply low CAD energy $(-3 \mathrm{~V})$ and high CAD energy $(-25 \mathrm{~V})$ conditions during ion accumulation. After ion accumulation and being transferred to the cell, all ions $(\mathrm{m} / \mathrm{z} 300-2000)$ were excited and detected with $256 \mathrm{~K}$ data points.

\section{Results and Discussion}

Large-scale data analysis of any type is often characterized by the rate of successful identification relative to the rate of false identification, such as false positive and false negative identification rates. ${ }^{13-15}$ This characterization is critical for large-scale science because large numbers of measurements are only useful if the false discovery rates are known or can be estimated. This report presents our efforts to develop novel informatics capabilities for our PIR projects and presents results on two separate components. These include useful estimates of false positive differentiation of PIR products and peptide identification strategies that allow initial estimation of PIR false discovery rates. We also present our efforts to develop an application, $X$-links, aimed at streamlining the data processing steps. Each of the identification factors, PIR product differentiation and peptide identification, was independently studied, and the false discovery rates were modeled for many thousands of hypothetical peptide masses as described below.

The PIR strategy is based on a unique class of chemical cross-linkers with reactive groups specific to primary amines or other functionalities and two labile bonds that can be fragmented with high specificity in the mass spectrometer. This allows one to cross-link proteins in intact 
cells and then, after the proteins are isolated and digested, one can measure cross-linked products and released peptide pairs by mass spectrometry. This stage of analysis is referred to as stage 2 analysis and is focused on the identification of PIR-labeled peptides and the differentiation of reaction product types. Stage 1 analysis is centered on the detection of PIRlabeled proteins, following affinity purification of PIR-labeled proteins, digestion, and shotgun 2D-LC-MS/MS analysis of all resulting peptides. Stage 1 analysis results in a restricted protein database focused on proteins from cells that are labeled by PIRs during our experiment. Stage 2 analysis is performed by first recording a precursor spectrum where the masses of potential PIR-labeled products may be present. Then, a second spectrum is recorded after low-energy CID activation is performed to cleave the labile PIR bonds, releasing the PIR reporter ion and previously labeled peptide ions. This pair of spectra is then analyzed to identify and differentiate the relationship between the masses in the precursor spectrum and those in the activation spectrum. These scan types, i.e., precursor and low-energy PIR activation scans, are alternated during LC separation of affinity purified PIR-labeled peptides. As a result, three possible PIR-labeled product types can be expected, namely, inter-cross-linked, intra-crosslinked, and dead-end labeled, which are illustrated in Figure 1, where $m_{\mathrm{p}}$ represents the precursor mass, $m_{\mathrm{r}}$ represents the reporter mass, and $m_{\mathrm{r} i}(i=1,2)$ indicates the released peptide masses. The resulting alternating spectral pairs acquired during LC-MS are processed to detect these relationships with the following basic steps:

1. All spectra are deisotoped using $I C R-2 L S .{ }^{16}$ This step results in a table (dataset) of neutral masses from each spectrum. The deisotoping algorithm implemented in $I C R-2 L S$ is based on THRASH procedure described by Horn et al. ${ }^{17}$

2. The datasets are segregated into two data streams that differentiate precursor and PIR activation scans, i.e., alternating spectra.

3. The spectra resultant from PIR activation scans are analyzed to determine if $m_{\mathrm{r}}$ is present. Upon finding reporter ions, these spectra are flagged to indicate that PIRrelevant products are present in these spectra.

4. The $m_{\mathrm{p}}$ values observed in precursor scans that are adjacent to flagged PIR activation scans from Step 3 are analyzed along with the fragmentation masses to see if any of the three expected PIR product types are present as illustrated in Figure 1. To detect $m_{\mathrm{r} i}$, every possible combination of masses in the fragmentation spectrum is searched for a match with any precursor mass. The following relationships are searched for in each pair of precursor and fragmentation spectra:

$$
\begin{aligned}
& \text { Inter }- \text { cross }- \text { link, } m_{\mathrm{p}}=m_{\mathrm{r} 1}+m_{\mathrm{r} 2}+m_{\mathrm{r}} \\
& \text { Inter }- \text { cross }- \text { link, } m_{\mathrm{p}}=m_{\mathrm{r} 1}+m_{\mathrm{r}} \\
& \text { Dead }- \text { end }, m_{\mathrm{p}}=m_{\mathrm{r} 1}+(\text { dead }- \text { end residual tag })+m_{\mathrm{r}}
\end{aligned}
$$

All possible matching products are then used for subsequent data processing and peptide identification. More than one PIR relationship can be present in a single spectrum thus supporting coelution of multiple PIR labeled products. We also place no requirement that the precursor $\left(m_{\mathrm{p}}\right)$ be absent in the activation spectrum or that the released peptides $\left(m_{\mathrm{r} i}\right)$ are absent in the precursor spectra.

Although it is possible for a released peptide to fall at the same mass as the PIR reporter, this fact is not used in the calculation of the false relationship discovery rate because this event is rare. As is shown in the Supporting Information Figure 1, only 1 PIR peptide is present in Shewanella oneidensis that matches the PIR reporter mass within 5 ppm MMA.

These steps form the basis of the application $X$-links that we have developed to provide automated PIR dataset processing. 
The high specificity in the PIR bond cleavage step results in unique tight search constraints for our analysis, because only PIR molecules are expected to fragment and produce the reporter ion during our analyses. To allow estimation of the rate at which one might expect PIR relationships to be falsely identified, a simulation was performed using the following procedures:

1. A set of peptide $m_{\mathrm{r} i}$ values was generated to simulate the results that are normally obtained following the deisotoping step of fragmentation spectral processing. These masses were randomly generated between the mass ranges of 500 and $5000 \mathrm{Da}$. This random list of masses was constructed by first generating random peptide sequences and then calculating the masses of the randomly generated peptides. The sequences were obtained from the Shewanella oneidensis MR-1 protein database by selecting random peptide sequences from the full set of proteins. The mass range used is consistent with the peptide masses detected in our mass analyzers. The number of masses generated represents the spectral complexity and is a parameter we varied to ascertain the effect on false relationship discovery. It is interesting to note that the use of truly random masses (i.e., non-peptide masses) resulted in lower false discovery rates (as can be seen in Figure 2 of the Supporting Information). This is as expected due to the fact that peptide masses tend to cluster around $1 \mathrm{Da}$ intervals as has been shown in the literature. ${ }^{18-20}$ This clustering results in the observation that the phase space covered by non-peptide random masses is much greater than peptide random masses and therefore results in a lower probability that 2 randomly chosen masses will sum to match a defined precursor mass.

2. Next, masses of 4002.97 and 1121.48 were chosen for precursor $\left(m_{\mathrm{p}}\right)$ and reporter ion $\left(m_{\mathrm{r}}\right)$ masses, respectively. The masses chosen represent actual peptide sequences and PIR molecular weights.

3. The precursor mass was used along with the peptide and reporter masses to identify potential PIR products. The mass measurement accuracy (MMA) was varied to allow determination of the impact of MMA on false relationship identification.

4. A Monte Carlo simulation was performed by selecting multiple sets of random peptide masses and repeating the analysis. Any cases other than the expected correct peptide masses found to sum together with the reporter mass to match the precursor mass were labeled as false discovery.

This simulation allows determination of the rate at which PIR products may be falsely identified.

The results of the false discovery of PIR product relationships are shown in Figure 2. In these results, the spectral complexity was varied from 20 masses $\left(m_{\mathrm{r} i}\right)$ to 100 masses $\left(m_{\mathrm{r} i}\right)$ in each hypothetical fragmentation spectrum. As mentioned above, a precursor mass $\left(m_{\mathrm{p}}\right)$ of 4002.97 Da was selected for this representation, but this procedure was performed using several different precursor masses with similar results. For each point on the plot in Figure 2, 10000 trials were performed and the number of false discovery relationships was recorded. These analyses were performed for mass measurement accuracy of 1, 5, and $10 \mathrm{ppm}$. In this case, mass measurement accuracy refers to the match between precursor masses and the summation of reporter and released peptide masses for the intercross-linked relationship as defined in Figure 1. These data illustrate the high degree of stringency of the PIR strategy and at $10 \mathrm{ppm}$ MMA and a fragmentation spectral complexity of 100 masses $\left(m_{\mathrm{ri}}\right)$, the false discovery rate is approximately $12 \%$. As mentioned above, this simulation is for the inter-cross-linking example and represents the most analytically challenging case due to the larger number of possible resultant combination masses from a given set of peptide masses. That is, intra-cross-linking and dead-end product identification require a less complex relationship with fewer possibilities 
and our simulations showed near zero false relationship detection for these product types as can been seen in Supporting Information Figure 3. In the case of inter-cross-linked product identification, two masses $\left(m_{\mathrm{r} 1}+m_{\mathrm{r} 2}\right)$ are needed to calculate the precursor mass. From combination theory it is known ${ }^{21}$ that selecting $k$ items (and allowing repeated selection) from a set of $n$ (masses, $\mathrm{m}_{\mathrm{ri}}$ ) has the following number of combinations:

Possibilities $=(n+k-1) ! /(k !(n-1) !)$

For a complexity of 100 masses $\left(m_{\mathrm{r} i}\right)$ and a set size of 2 , this yields a total of 5050 possibilities compared with only 100 possibilities for the intra-cross-link and dead-end products. Thus, the false discovery rates for inter-cross-link relationship rates are conservative estimates for all PIR product false discovery rates.

\section{Peptide Identification Strategies}

After the peptide relationships have been detected, the masses $\left(m_{\mathrm{r} i}\right)$ and the constraints imposed by this PIR strategy are used to search an organism database to identify the peptide sequence and protein or proteins that contain the peptide. The peptide identification search is further constrained by the requirement that each peptide contain an internal (non-terminal) lysine. This is required for cross-linking reaction because the current PIRs cross-linkers are primary aminereactive. The following procedure was used to perform this analysis:

1. The Shewanella oneidensis MR-1 database of predicted proteins was used to calculate a list of all tryptic peptides with up to 1 missed cleavage and an internal lysine.

2. This list of peptide sequences was then used to calculate the exact mass of each peptide.

3. Using each mass $\left(m_{\mathrm{r} i}\right)$ in this list, we searched the entire list with varying mass measurement accuracies to determine the uniqueness of each mass $\left(m_{\mathrm{r} i}\right)$ entry in the list.

As mentioned above and described in detail elsewhere, ${ }^{12}$ stage 1 analysis results in a restricted protein database with lower complexity in terms of the possible proteins and peptides. This experiment produces a restricted list of proteins that are identified by conventional LC-MS/ MS proteomics measurements to be PIR-labeled in on-cell labeling experiments. For example, from $S$. oneidensis, about 400 proteins were identified in stage 1 experiments. ${ }^{22}$ This experimentally developed stage 1 database was also used to repeat the above calculations and to illustrate its impact on the uniqueness of peptide identification.

The results of these uniqueness calculations can be seen in Figure 3. The upper plot in this figure (Figure 3a) shows the results from the full Shewanella oneidensis MR-1 database. In this case, a total of 67507 peptides were calculated using X-links and the full Shewanella oneidensis MR-1 database. This list contained all the peptides with an internal lysine and up to 1 missed tryptic cleavage site. Figure 3 a shows three different curves. The " 1 possibility" curve is the case where the peptide mass is uniquely identified in the database (i.e., the peptide mass matches only one peptide sequence found in the database) at the level of mass measurement accuracy (MMA) shown on the X-axis. The "2 possibilities" curve is the case where searching the database for the detected mass $\left(m_{\mathrm{ri}}\right)$ results in 2 or fewer peptide sequences at a given MMA and the " 3 possibilities" curve is the case where the database search results in three or fewer peptides that could have produced the searched mass. It is noteworthy that even with no mass measurement error, i.e., search of the database with exactly matching masses; $80 \%$ of the peptide masses that satisfy the PIR constraints are unique. This peptide uniqueness increases to $87 \%$ with up to two peptide matches and $89 \%$ with up to 3 possible matches. In addition, even if its not possible to uniquely identify a peptide, the utility in identification of a set of 2 or 3 peptides as part of a detected protein interaction can be further 
resolved with additional mass spectrometry information, antibody-based experiments, and other published results. For example, additional peptide identification data and other biological information could be used to remove the ambiguity for relevant cases. Finally, an additional spectrum can be collected after PIR activation that also includes further activation (such as IRMPD or $\mathrm{CID}^{23,24}$ ) to fragment the peptide products released during the low energy activation step. This process will create fragments for the released peptides and the reporter ion. Masselon et al. ${ }^{25}$ previously showed that with as few as 3 fragments and 5 ppm mass measurement error, $98 \%$ of the tryptic peptides can be uniquely identified using this multiplexed MS/MS strategy on complex prokaryotic organisms. Fragment ions resultant from the CID or IRMPD activation of the reporter ion itself can also be used as internal calibrants to enable post-experiment calibration of each spectrum. This will readily allow 5 ppm MMA.

The ability to uniquely identify a peptide using accurate mass is a function of the database complexity as can be seen in Figure $3 \mathrm{~b}$. In this case, the percentage of unique peptides is calculated from the MR-1 restricted protein database resulting from the stage 1 analysis. This database contains only 367 proteins compared to 4854 in the full database and includes all proteins that were positively identified as PIR-labeled products. This reduced complexity results in 8520 peptides compared to the 67507 for the full database. It is interesting to note that this reduction in number of peptides by 7.9 results in an increase in the percentage of unique peptides from 80.3 to $94.1 \%$, an increase in the percentage of unique peptides by a factor of only 1.17; this relationship is a strong function of MMA as can be seen in Figure 3a and $3 \mathrm{~b}$. These data indicate that the percentage of unique peptides is not a linear function of the peptide complexity; thus, increasing the complexity by an order of magnitude will not reduce the percentage of unique peptides by an order of magnitude. The $X$-links application was used to calculate the data used for this unique peptide analysis. The PIR strategy false discovery rate is limited by this peptide unique identification limit. The calculation of unique peptides can be used to determine an estimate of the minimum false discovery rate. This is accomplished by calculating the number of peptides that can be uniquely identified by mass in the database and then calculating the number of cases where the database search results in 2 peptides that match the search mass $\left(m_{\mathrm{r} i}\right)$, and next the number of cases where searching the database results in 3 peptides that match the search mass. This procedure was repeated for all peptide masses in the database. These data were then used, at each mass measurement accuracy, to estimate the percentage of peptides masses that will be falsely identified using accurate mass alone. These results were then combined to estimate a minimum false discovery rate for the mass assignment to a peptide sequence. For example, if we let $P i$ equal the number of peptides that match $i$ times in the database where $i$ varies from 1 (unique match) to the maximum number of peptide matches for any peptide mass in the database, the total false discovery percentage can then be calculated as:

False discovery percentage $=$

$$
\left(1-\left(\sum_{i=1}^{i=\max } P i / i\right) /(\text { Total peptide count in database })\right) 100
$$

This calculation is performed at various mass measurement accuracy values using both the full Shewanella oneidensis database and the stage 1 reduced database as shown in Figure 4. This false discovery calculation is an estimate of the likelihood that a peptide measured at a given MMA and a given database complexity will be uniquely identified in a search of the database. These calculations were performed using peptide masses in the range of 500 to $5000 \mathrm{Da}$ and represent a composite rate for this mass range.

The approach used here to develop the false discovery rate differs from the use of a reverse database search that has been extensively applied ${ }^{26-28}$ to analysis of tandem MS/MS results. In the present case, we are searching the database for accurate peptide mass matches and the database complexity will define the level of false discovery, regardless of sequence order. In 
any event, the procedure described above was also performed using a reverse MR-1 database to ascertain the effect on false discovery rates. The results of this reverse database search were virtually identical with the forward database, as can be seen in Figure 4 of the Supporting Information, suggesting reverse database search is redundant with false discovery rate estimates above.

\section{X-links Application}

The development of a PIR data processing application ( $X$-links) was motivated by the unique needs of the PIR strategy and the fact that the necessary tools are not available. The PIR strategy utilizes high mass measurement accuracy and sequence constraints to identify peptides while other strategies perform identification utilizing peptide backbone fragmentation data and/or stable isotope labeling. $9,11,29,30$ The data processing starts with raw mass spectra mass transformation or deisotoping using the ICR-2LS application ${ }^{16}$ developed at PNNL which generates a list of neutral masses representing all the peptide features detected in the spectrum. This list of neutral masses is saved in a PEK file. X-links uses the output from ICR-2LS and loads the content of the PEK files into a data array. The user interacts with $X$-links through a familiar Windows graphical user interface paradigm; the interface is shown in Figure 5. $X$ links has been developed to support PIR research by processing the de-isotoped PEK files that result from the ICR-2LS analysis of the raw mass spectra. $X$-links contains a number of features to aid in this PIR data processing including support for simulations discussed in this paper.

Below is a list of a few of the features built into the $X$-links application:

- $\quad$ provides visual and analytical tools for evaluation of low and high-resolution mass spectra deconvoluted to a list of masses.

- $\quad$ provides interface to spectrum-level summaries. These summaries include data such as the total count of isotopic distributions detected, isotopic distribution abundance data, and time domain signal level data.

- allows inspection of various data attribute distributions. These distributions include histograms developed to aid in the assessment of dataset quality and data richness by illustrating distributions of masses, isotopic fits or quality factors, abundances, peptide masses, protein masses, and peptides matches.

- allows visual interactive abundance and elution profiling of selected masses.

- $\quad$ provides extensive and flexible in-silico fasta file calculation and inspection including peptide digestion based on user defined cleavage rules.

- allows matching between experimental data and generated peptide lists.

- $\quad$ provides interface for automated detection of PIR products and annotation of possible peptide identifications from the selected features.

- $\quad$ provides functions allowing simulation of PIR detection process and estimate of its false discovery rates. The false discovery rates for peptide identification as well as PIR product are calculated and presented. Future versions of $X$-links will combine these two rates to calculate an overall false discovery rate.

The $X$-links application exploits the high resolution and accurate mass capability of FTICRMS but features have been added to support lower performance mass analyzers. For example, Mascot Generic Format (MGF) files can be loaded in X-links as well. In the case of lowresolution data that has not been deisotoped, a charge state must be assumed to complete the analysis however. This assumption will contribute to the ambiguity of assignments; therefore, $X$-links is primarily designed for high-resolution data. Multiple data filters are available to the user when loading high-resolution deisotoped data from PEK files. The PEK files contain lists of all detected features in the mass spectra. This list includes the deisotoped neutral masses 
and a number of other parameters including the ion or "feature" signal strength and a feature quality factor. These filters allow the user to load selected data from a PEK file. This can include a user-defined mass range of interest and selection of data features with user defined signal strength and quality factor.

$X$-links implements and automates the PIR strategy described above and elsewhere. ${ }^{8}$ The functionality of this software allows searching for potential PIR cross-linked products in consecutive spectra and reporting the final results in an output data file. PIR-specific information is stored in an initialization file allowing easy incorporation of newly engineered PIR reagents. This information includes the mass of the reporter ion and the mass change for each peptide resulting from cleavage of the labile bonds. This mass change is a result of the PIR molecule reaction with the primary amine of the cross-linked peptide. After the labile bonds are broken and the peptides are released, the chemical modification to the peptides results in a mass change. $X$-links uses this mass change information to calculate the unmodified peptide mass, thus allowing identification.

$X$-links provides functions to allow the automated analysis of PIR datasets generated using FTICR or other mass spectrometers. These files are first deisotoped using ICR-2LS resulting in PEK files that $X$-links loads for PIR detection. The algorithm implemented in $X$-links is shown in Figure 6. This algorithm performs the following steps:

1. The spectra are arranged in the dataset with the low energy activation spectra following the precursor spectra. The procedure starts with the low energy activation spectra. A dataset could consist of several hundred to thousands of spectra.

2. The first test performed is inspection of the activation spectrum to confirm the presence of a reporter ion. If this ion is found, the analysis is continued with the next step. Otherwise the program advances to the next pair of precursor/activation spectra.

3. After detection of a reporter ion, $X$-links then uses the masses found in the precursor spectrum and the masses in the activation spectrum $\left(m_{\mathrm{r}}, m_{\mathrm{r} i}\right)$ to locate and PIR product relationships. $X$-links allows the user to define tolerances used for this relationship detection and reporting.

4. The reported findings, including important mass error distribution statistics for reporter and product ions, are output on a separate worksheet.

This process is repeated for all spectra in an mass spectrometry dataset. The resulting data represents a significant reduction in data complexity with a high degree of confidence that the detected relationships represent cross-linked peptides. After the PIR relationship detection has been completed, $X$-links will search an organism database to identify the protein of origin for each detected peptide. Identified peptides and a list of potential cross-linked proteins are included in this final report.

$X$-links contains additional diagnostic tools used to evaluate the performance of the mass spectrometer dataset. These tools include the ability to develop error histograms as shown in Figure 7. These histograms illustrate the instrument's mass measurement performance by analyzing the detected reporter ion mass. This analysis allows the calculation of error histograms for the PIR product ions, and this information is critical in determination of the MMA level that should be used in subsequent database searches using the PIR product masses. These error histograms illustrate the mass spectrometers performance in terms of MMA and in measurement repeatability. The histograms can be used as a flag to indicate the need for post-experiment calibration and then for setting the MMA value used for the database search. Additional quality control algorithms are being developed to utilize the elution profiles of the product and reporter ions. The elution profiles of the released peptides (observed in the activation scans) and the associated precursor ions (observed in the precursor scans) should 
show a high degree of correlation, since all ions are derived from the same species. Later versions of $X$-links will utilize this feature to help further automated data processing and provide an additional quality control step. This feature will add in the determination of PIR product peptides and support the analysis of complex datasets by providing a way to identify PIR products in the case of elution of overlapping cross linked products.

The utility of this error histogram feature can be seen in Figure 7a) shows the error histograms for the 1+ and 2+ charge states of the PIR report ion. In an ideal (perfect calibration) case these two histograms would overlap and both be centered around $0 \mathrm{ppm}$ error. As can been seen, the $1+$ ions have a negative error and the $2+$ ions a positive error. (Figure $7 \mathrm{~b}$ ) shows the impact of post calibration of the mass spectrometer using the PIR mass $\left(m_{\mathrm{r}}\right)$ as an internal lock mass. After internal calibration, the two distributions overlap and are centered around $0 \mathrm{ppm}$ error.

To demonstrate the utility of $X$-links, a sample cross-linked pair of peptides were analyzed and the results are shown in Figure 8. In this case, bradykinin and neurotensin peptides were reacted with the PIR cross-linking molecule to create a known complex. This sample of dead-end, inter and intra-cross-linked peptides was then analyzed with ESI-FTICR-MS. The top spectrum in this figure was acquired with low collision energy (collision cell voltage $=-3 \mathrm{~V}$ ) to allow detection of intact PIR-peptide complexes. The inset in this spectrum illustrates a PIR-labeled neurotensin-bradykinin pair of peptides. The bottom spectrum was acquired with higher collision energy (collision cell voltage $=-25 \mathrm{~V}$ ) to promote PIR fragmentation and release of peptides. The insets illustrate the reporter ion $\left(m_{\mathrm{r}}=841\right)$, the two released peptides $\left(m_{\mathrm{r} 1}=1770\right.$, $m_{\mathrm{r} 2}=841$ ), and the observed mass measurement error. These data were then processed by $X$ links to illustrate its ability to detect and identify PIR product. Figure $8 \mathrm{c}$ illustrates $X$-links output analysis of these two spectra.

The $X$-links application is freely available for download at http://www.wsu.edu/proteomics/. A brief description of functionalities and usage is available in an included PowerPoint presentation. ICR- $2 L S$ software is also freely available for download from http://ncrr.pnl.gov/. To verify the assignments of cross-linked products, we used Mascot for annotation of high-energy MS/MS spectra.

\section{Conclusion}

The $X$-links application provides a new platform required to enable PIR research in an automated and high throughput fashion. $X$-links encompasses a suite of tools to enable the analysis of the PIR datasets and report the cross-linked peptides and proteins that are indicated by the relationships found in the datasets. Additionally, the described false discovery rate determination provides a practical method for estimation of peptide identification confidence. This false discovery estimation is an additional feature included in X-links.

The reporter ion detected in the low energy activation spectrum plays a critical role in the PIR strategy. Not only does this reporter flag spectra indicating that product ions are present, it also provides an internal calibration peak that can be used to post calibrate spectra. This internal calibration peak is used as a lock mass to perform a one point calibration adjustment. 31,32 Given the importance of MMA on the false discovery rates, this step will allow low to subppm levels of performance. The precursor spectra can also be calibrated indirectly using this reporter ion mass. After the activation spectrum is recalibrated, any masses that are detected in both the precursor spectrum and the recalibrated activation spectrum can be used as internal standards to improve the precursor MMA.

\section{Supplemental Materials}

Refer to Web version on PubMed Central for supplementary material. 


\section{Acknowledgements}

Sequence data for Shewanella oneidensis MR-1 was obtained from The Institute for Genomic Research website at http://www.tigr.org. A portion of the research described in this paper was was performed in the Environmental Molecular Sciences Laboratory (EMSL), a national scientific user facility sponsored by the Department of Energy's Office of Biological and Environmental Research and located at the Pacific Northwest National Laboratory. We acknowledge help and services of High Performance Computing \& Networking Services group at EMSL and IT personal at Department of Chemistry at WSU for enabling safe and transparent sharing and flow of data between EMSL and WSU. This research was supported by Office of Science (BER), U.S. Department of Energy. Grant No. DE-FG02-04ER63924.

\section{References}

1. Yao T. Bioinformatics for the genomic sciences and towards systems biology. Japanese activities in the post-genome era. Prog Biophys Mol Biol 2002;80(1-2):23-42. [PubMed: 12231221]

2. Anderson NG. Co-immunoprecipitation. Identification of interacting proteins. Methods Mol Biol 1998;88:35-45. [PubMed: 9664296]

3. White MA. The yeast two-hybrid system: forward and reverse. Proc Natl Acad Sci USA 1996;93(19): 10001-10003. [PubMed: 8816737]

4. Zanetti G, Merati G. Interaction between photosystem I and ferredoxin. Identification by chemical cross-linking of the polypeptide which binds ferredoxin. Eur J Biochem 1987;169(1):143-146. [PubMed: 2824198]

5. Sinz A, Kalkhof S, Ihling C. Mapping protein interfaces by a trifunctional cross-linker combined with MALDI-TOF and ESI-FTICR mass spectrometry. J Am Soc Mass Spectrom 2005;16(12):1921-1931. [PubMed: 16246579]

6. Peterson JJ, Young MM, Takemoto LJ. Probing alpha-crystallin structure using chemical cross-linkers and mass spectrometry. Mol Vis 2004;10:857-866. [PubMed: 15570221]

7. Bennett KL, et al. Chemical cross-linking with thiol-cleavable reagents combined with differential mass spectrometric peptide mapping-a novel approach to assess intermolecular protein contacts. Protein Sci 2000;9(8):1503-1518. [PubMed: 10975572]

8. Tang X, et al. Mass spectrometry identifiable cross-linking strategy for studying protein-protein interactions. Anal Chem 2005;77(1):311-318. [PubMed: 15623310]

9. El-Shafey A, et al. Zero-length" cross-linking in solid state as an approach for analysis of proteinprotein interactions. Protein Sci 2006;15(3):429-440. [PubMed: 16501223]

10. Chowdhury SM, et al. Collisionally activated dissociation and electron capture dissociation of several mass spectrometry-identifiable chemical cross-linkers. Anal Chem 2006;78(24):8183-8193. [PubMed: 17165806]

11. Chen T, Jaffe JD, Church GM. Algorithms for identifying protein cross-links via tandem mass spectrometry. J Comput Biol 2001;8(6):571-583. [PubMed: 11747613]

12. Tang X, et al. Profiling the membrane proteome of Shewanella oneidensis MR-1 with new affinity labeling probes. J Proteome Res 2007;6(2):724-734. [PubMed: 17269728]

13. Cargile BJ, Bundy JL, Stephenson JL Jr. Potential for false positive identifications from large databases through tandem mass spectrometry. J Proteome Res 2004;3(5):1082-1085. [PubMed: 15473699]

14. Huttlin EL, et al. Prediction of error associated with false-positive rate determination for Peptide identification in large-scale proteomics experiments using a combined reverse and forward Peptide sequence database strategy. J Proteome Res 2007;6(1):392-398. [PubMed: 17203984]

15. Xie H, Griffin TJ. Trade-off between high sensitivity and increased potential for false positive peptide sequence matches using a two-dimensional linear ion trap for tandem mass spectrometry-based proteomics. J Proteome Res 2006;5(4):1003-1009. [PubMed: 16602709]

16. Anderson, GA. ICR-2LS, FTICR data visulization and analysis application. Pacific Northwest National Labratory; 1994.

17. Horn DM, Zubarev RA, McLafferty FW. Automated reduction and interpretation of high resolution electrospray mass spectra of large molecules. J Am Soc Mass Spectrom 2000;11(4):320-332. [PubMed: 10757168] 
18. Clauser KR, Baker P, Burlingame AL. Role of accurate mass measurement (+/- $10 \mathrm{ppm})$ in protein identification strategies employing MS or MS/MS and database searching. Anal Chem 1999;71(14): 2871-2882. [PubMed: 10424174]

19. Conrads TP, et al. Utility of accurate mass tags for proteome-wide protein identification. Anal Chem 2000;72(14):3349-3354. [PubMed: 10939410]

20. Spengler B. De novo sequencing, peptide composition analysis, and composition-based sequencing: a new strategy employing accurate mass determination by fourier transform ion cyclotron resonance mass spectrometry. J Am Soc Mass Spectrom 2004;15(5):703-714. [PubMed: 15121200]

21. Graham, RL.; Grötschel, M.; Lovász, L. Handbook of Combinatorics. 1 and 2. Elsevier; NorthHolland, Amsterdam: MIT Press; Cambridge, MA: 1996.

22. Yates JR 3rd, Eng JK, McCormack AL. Mining genomes: correlating tandem mass spectra of modified and unmodified peptides to sequences in nucleotide databases. Anal Chem 1995;67(18):3202-3210. [PubMed: 8686885]

23. Little DP, et al. Infrared multiphoton dissociation of large multiply charged ions for biomolecule sequencing. Anal Chem 1994;66(18):2809-2815. [PubMed: 7526742]

24. Wu Q, et al. Characterization of cytochrome $\mathrm{c}$ variants with high-resolution FTICR mass spectrometry: correlation of fragmentation and structure. Anal Chem 1995;67(14):2498-2509. [PubMed: 8686880]

25. Masselon C, et al. Accurate mass multiplexed tandem mass spectrometry for high-throughput polypeptide identification from mixtures. Anal Chem 2000;72(8):1918-1924. [PubMed: 10784162]

26. Nesvizhskii AI, et al. A statistical model for identifying proteins by tandem mass spectrometry. Anal Chem 2003;75(17):4646-4658. [PubMed: 14632076]

27. Sadygov RG, Cociorva D, Yates JR 3rd. Large-scale database searching using tandem mass spectra: looking up the answer in the back of the book. Nat Methods 2004;1(3):195-202. [PubMed: 15789030]

28. Sadygov RG, Liu H, Yates JR. Statistical models for protein validation using tandem mass spectral data and protein amino acid sequence databases. Anal Chem 2004;76(6):1664-1671. [PubMed: 15018565]

29. de Koning LJ, et al. Computer-assisted mass spectrometric analysis of naturally occurring and artificially introduced cross-links in proteins and protein complexes. FEBS J 2006;273(2):281-291. [PubMed: 16403016]

30. Gao Q, et al. Pro-CrossLink. Software tool for protein cross-linking and mass spectrometry. Anal Chem 2006;78(7):2145-2149. [PubMed: 16579592]

31. Burton RD, et al. Exact Mass Measurements Using a 7 Tesla Fourier Transform Ion Cyclotron Resonance Mass Spectrometer in a Good Laboratory Practices-Regulated Environment. Am Soc Mass Spectrom 1999;10(12):1291-1297.

32. Duan L, Chan TW. A modified internal lock-mass method for calibration of the product ions derived from sustained off-resonance irradiation collision-induced dissociation using a Fourier transform mass spectrometer. Rapid Commun Mass Spectrom 2004;18(12):1286-1294. [PubMed: 15174182] 


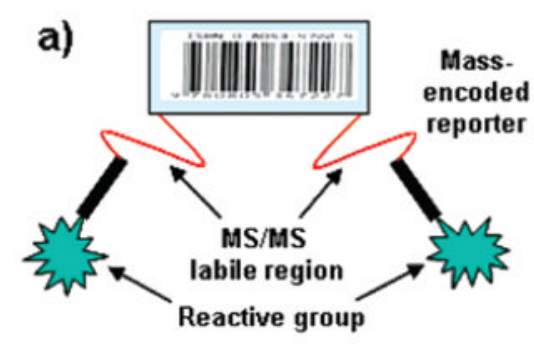

\section{Protein Interaction Reporter (PIR) Strategy}

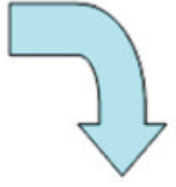

Low energy CID

activation

b)

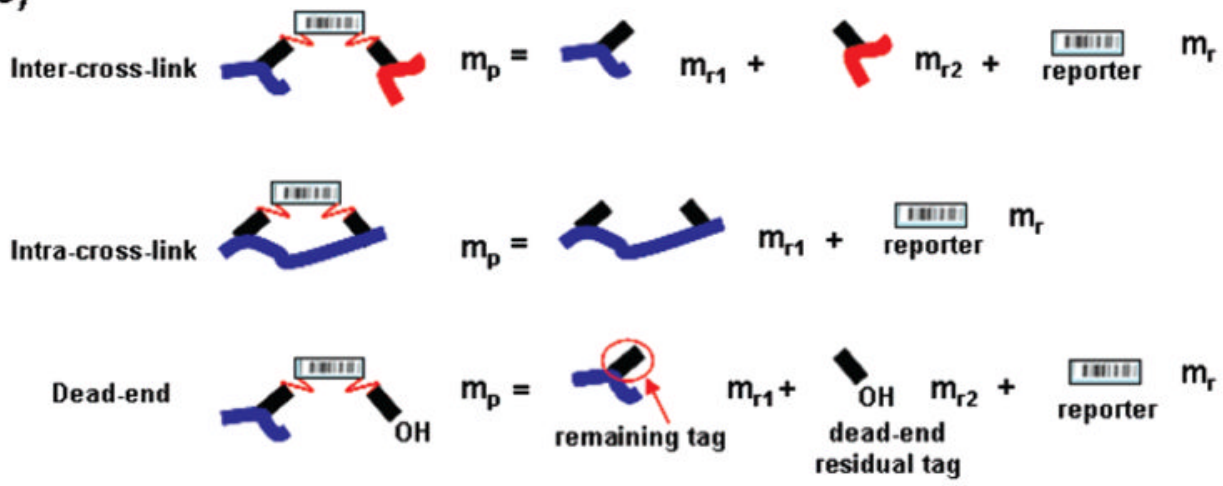

Figure 1.

(a) Features of conceptual protein interaction reporter structure. (b) The specific fragmentation pattern of PIR-labeled peptides helps distinguish dead-end, intra-, and inter-cross-linked peptides. The neutral mass of the precursor ion equals the sum of the neutral masses of its product ions. 


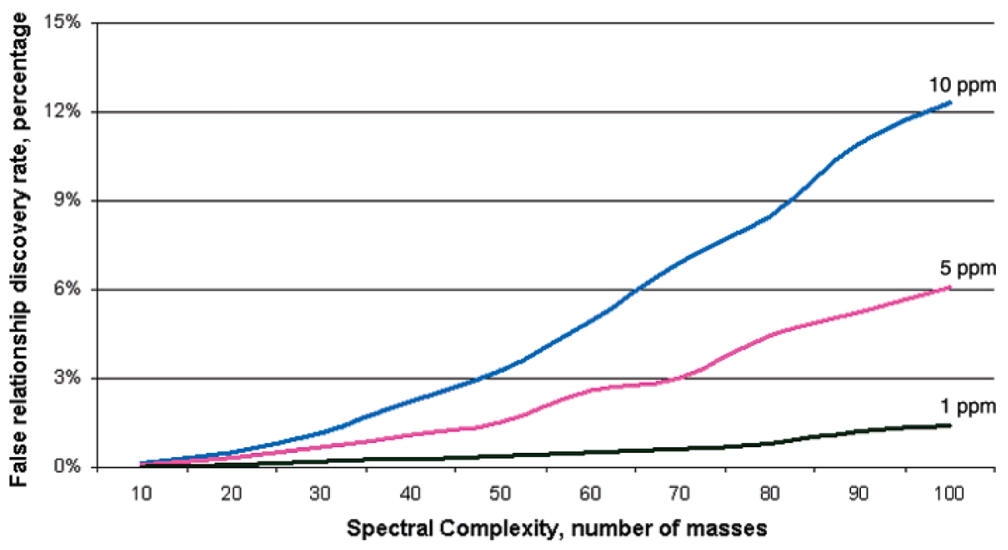

Figure 2.

False PIR mass relationship discovery rate in percentage. This analysis was performed for the inter-cross-link products that represent the most challenging informatics analysis case. These data also illustrate the impact of spectral complexity and instrument mass measurement accuracy (MMA) on the false relationship discovery rate. 
Peptide uniqueness using Shewanella oneidensis MR1 - Tryptic Peptides Internal Lys
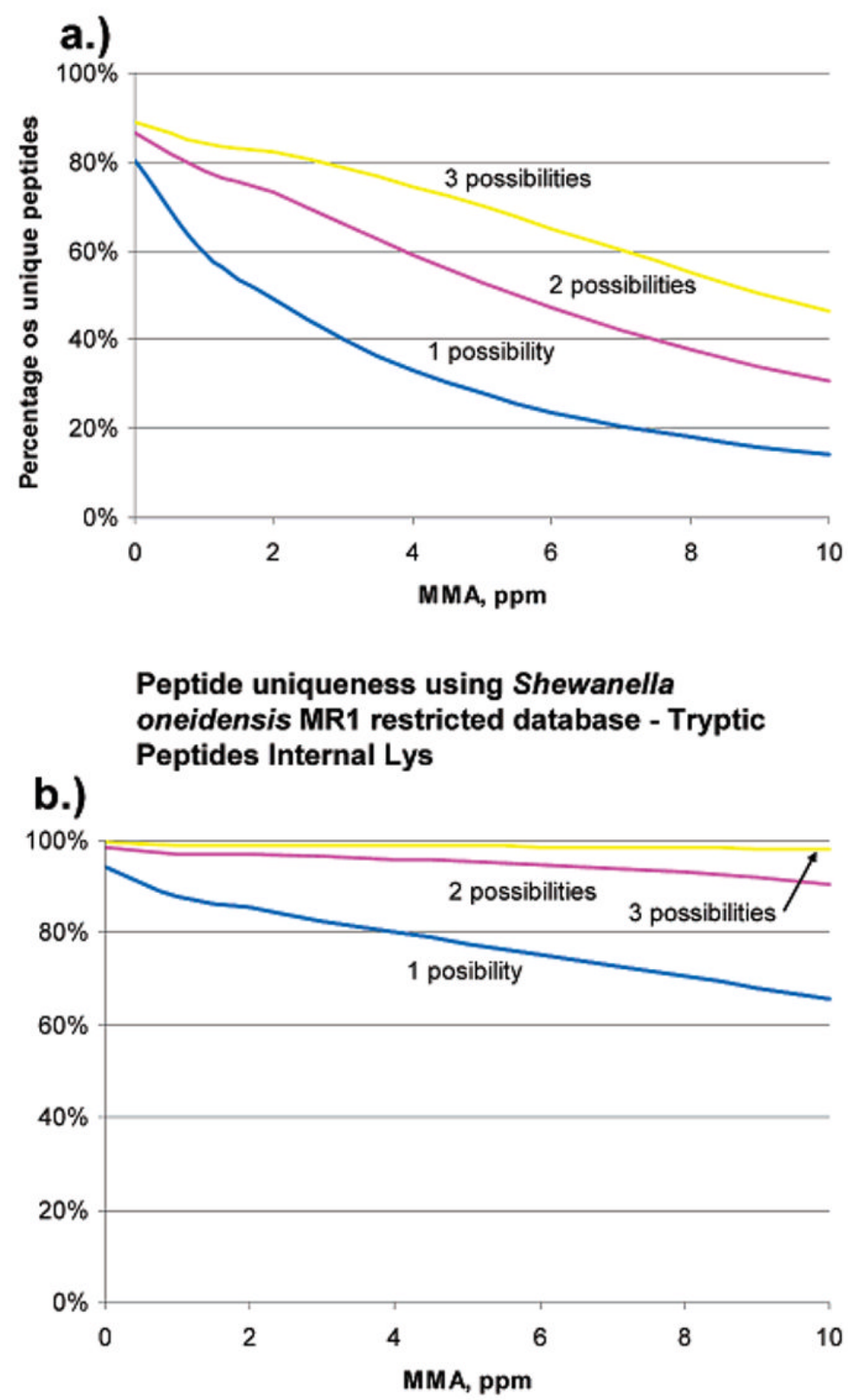

Figure 3.

Percentage of unique peptides of Shewanella oneidensis using both the full protein database and the stage 2 restricted database. (a) was developed using the full database resulting in 67 507 peptides. (b) is from the restricted database with 8520 peptides. All peptides contain an internal lysine. Three curves are presented in each plot: "1 possibility" represents the case where database search results in only a single peptide being identified, "2 possibilities" is the case where 1 or 2 peptides are identified, and " 3 possibilities" is the case where 1, 2, or 3 peptides are identified. 


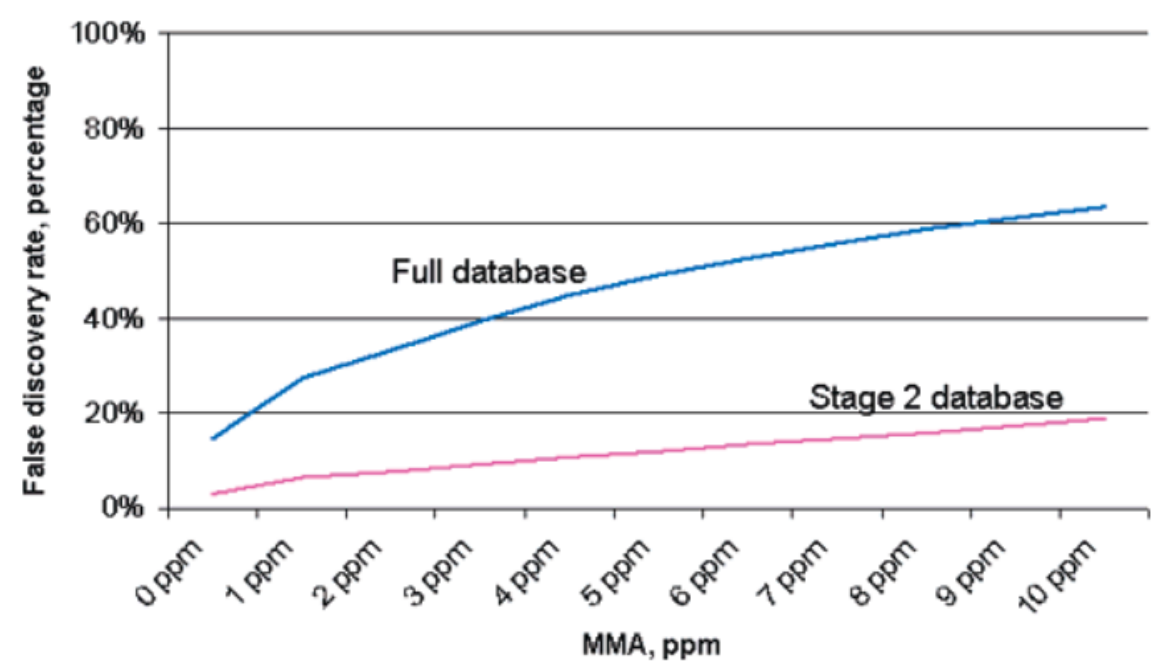

Figure 4.

False discovery rate estimates for Shewanella oneidensis using both the full protein database and the restricted database of proteins found to be labeled by PIRs. These data show that with modest MMA and a reduced database the likelihood of uniquely identifying a PIR product ion is high $(>90 \%)$. 


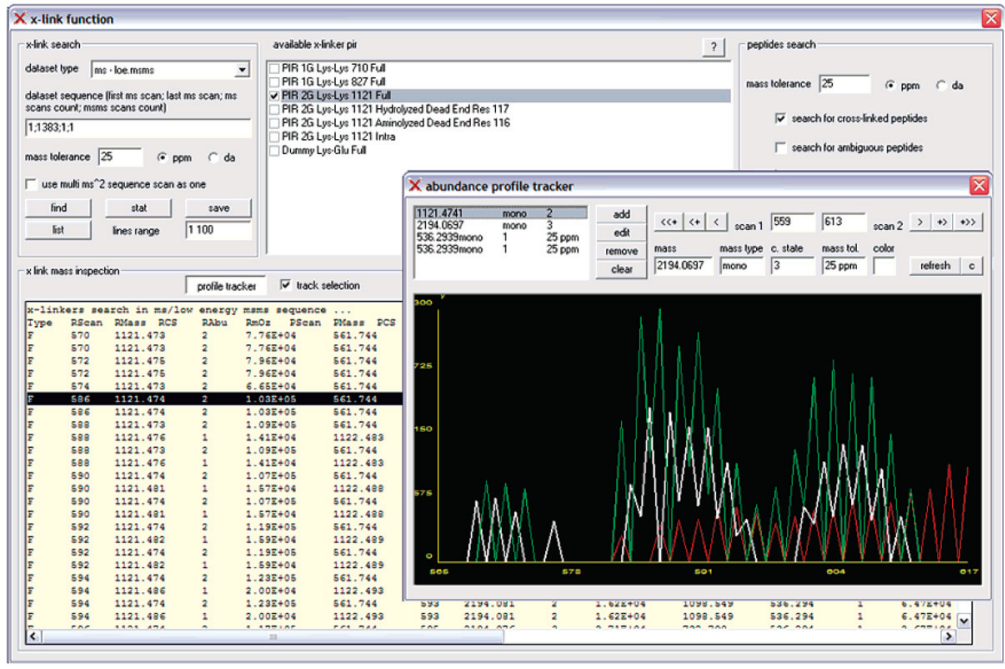

Figure 5.

$X$-link screenshot illustrating the most important functionalities of the software. Dialog box in upper left corner shows the user interface for cross-link product identification function. Output results are presented in standardized fashion in separate worksheet on bottom. Chart on the right presents total ion and extracted ion chromatograms that are generated automatically by $X$-links from the loaded dataset as an illustration of charting capabilities. 


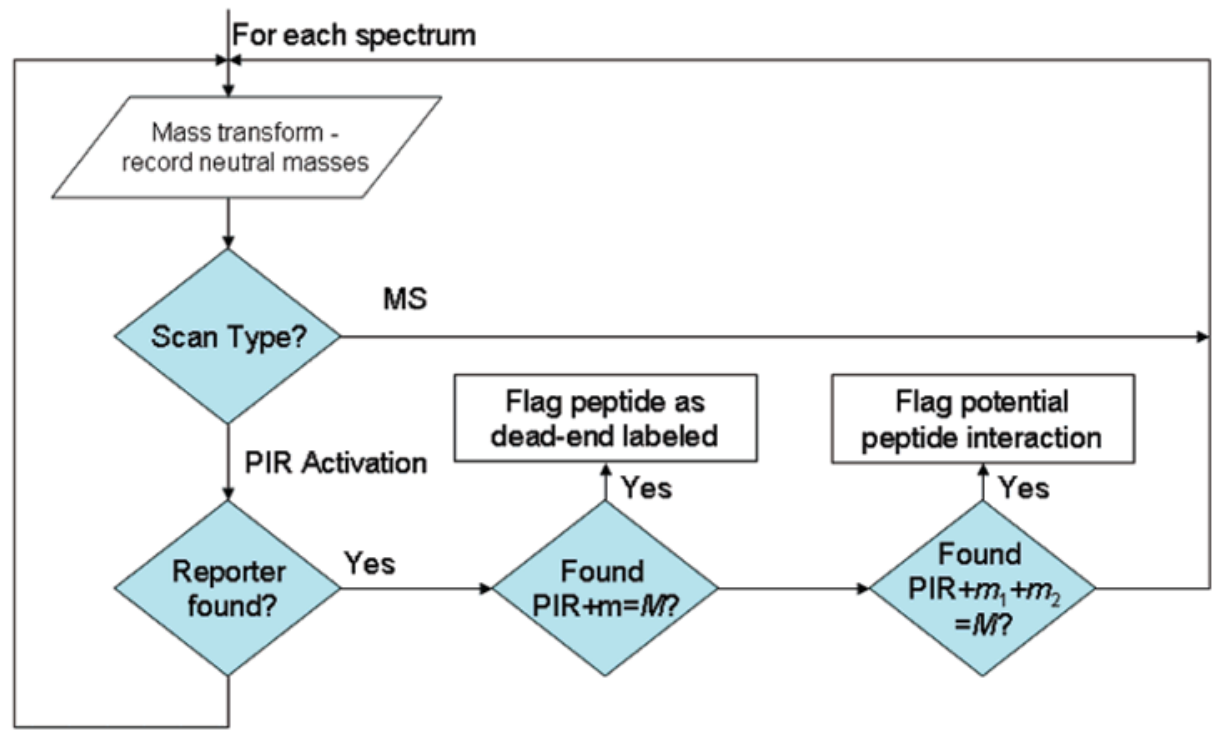

Figure 6.

Flowchart for $X$-links putative cross-link product detection in PIR based LC-MS/low-energy dissociation MSMS experiment ( $m$ indicates a mass from PIR activation spectra, $M$ indicates a mass derived from PIR precursor spectra). 

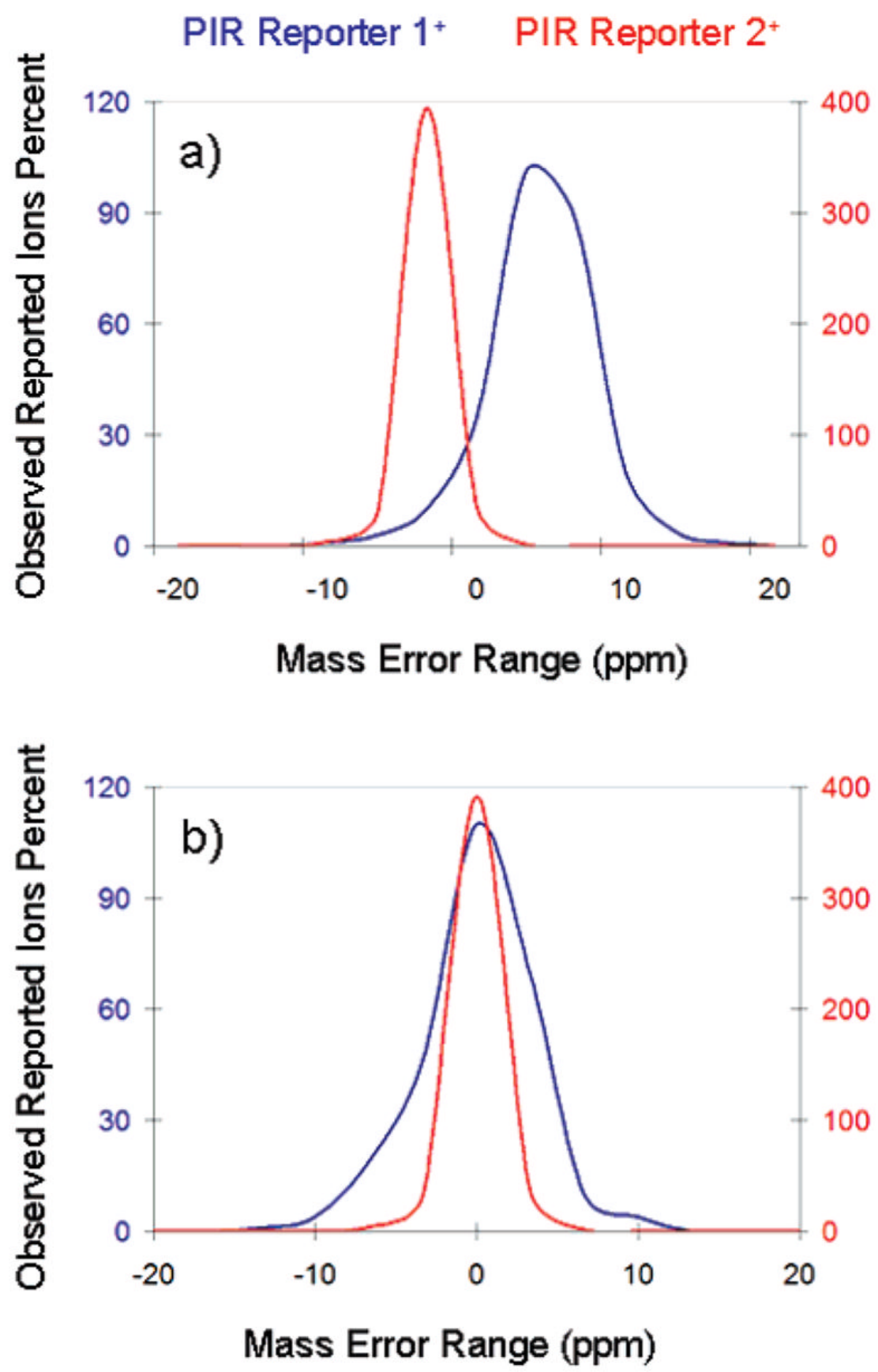

Figure 7.

(a) Mass measurement error distributions for 1+ and 2+ PIR reporter ions prior to internal calibration. (b) Mass measurement accuracy improvements resultant from use of PIR reporter ion as an internal calibrant. 
a)

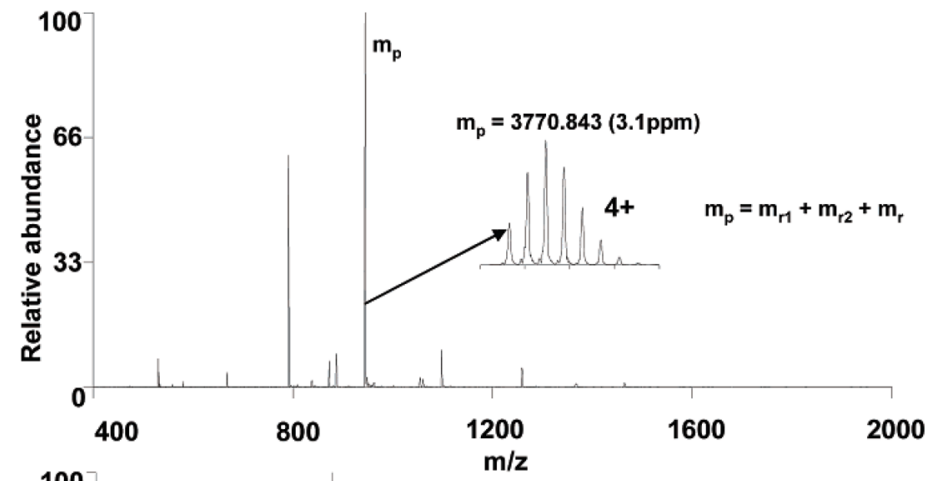

b)

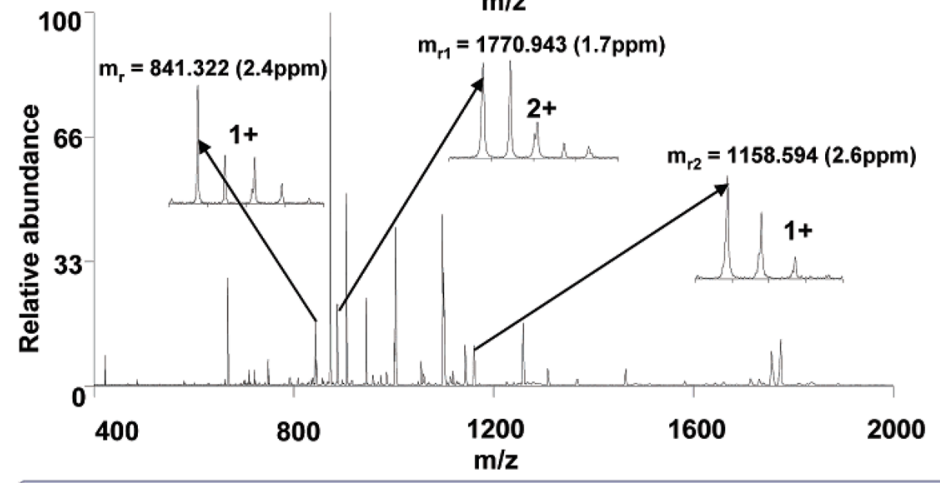

c)

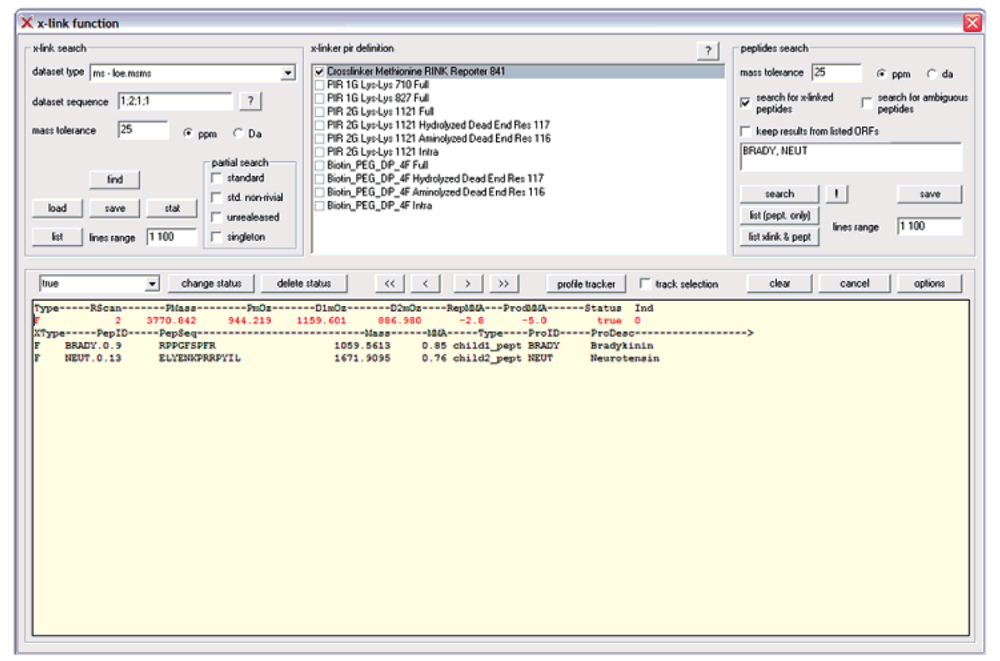

Figure 8.

Example PIR dataset of cross-linked neurotensin and bradykinin. (a) Low-energy spectrum (collision cell voltage $=-3 \mathrm{~V}$ ) with the $m_{\mathrm{p}}$ ion identified by $X$-links. (b) PIR activation spectrum (collision cell voltage $=-25 \mathrm{~V}$ ) and the released peptides and the reporter ions. $m_{\mathrm{p}}$ is the sum of the two peptide masses ( $m_{\mathrm{r} 1}$, neurotensin and $m_{\mathrm{r} 2}$, bradykinin) plus the reporter ion mass, $m_{\mathrm{r}}$. The two peptides masses are modified by the expected residual fragment of the PIR crosslinker (99.03) resulting in calculated neutral peptide masses neurotensin $=1770.940$, bradykinin $=1158.591$ and the reporter mass $=841.324$. These masses total to an expected neutral precursor mass of 3770.855 . The expected values match well with measured values and the observed mass measurement errors in PPM are shown on the figure. (c) Screen capture image of the program $X$-links output resultant from analysis of the spectra in (a) and (b). $X$ links successfully identified the expected cross-linked product by comparison of summed neutral masses observed in spectrum (b) to the measured neutral masses in spectrum (a). The 
inter-linked product between neurotensin and bradykinin was identified and listed as a product type "F" which indicates full or inter-cross-linked product between the two peptides. 